E reiform-Datenbank“ - hat weder - etwas mit einer Kür zu tun noch einem Knigge für Alt-68er. Die auch im Englischen - holprige Bezeichnung des Software-Klassikers askSam versucht zwei Grundeigenschaften dieses Programms zu umreißen: Es handelt sich um eine Datenbank - vor allem in Hinsicht auf die Geschwindigkeit - ohne typische Begrenzungen von Datenbankstrukturen. Kurzum: askSam-Anwender füllen Ihre Informationen - woher auch immer diese stammen - in eine Datei und können dann blitzschnell alle Informationen durchsuchen, verknüpfen, editieren, Reports erstellen oder drucken. Angesichts der enormen Funktionsfülle bereits der älteren Versionen von askSam erscheinen die neuen Eigenschaften der gerade erschienen Version 6 wie ein Sahnehäubchen auf einer Schwarzwälder Kirschtorte.

\section{Web-Archivierung leicht gemacht}

Quellen einer askSam-Datenbank können sein: Adobe PDF-Dateien, unformatierte Text-Dateien (z.B. ASCII), Texte im Austauschformat RTF, HTML-Dateien (siehe weiter unten), Texte typischer Textverarbeitungsprogramme (Microsoft Word, WordPerfect u. a.), E-Mails aus allen Standardprogrammen (Microsoft Outlook, Eudora, PocoMail, Netscape etc.), Datenbank-Informationen (z.B. aus Microsoft Access, dBASE, Paradox, ODBC), Tabellenkalkulationsdateien (Microsoft Excel), alle standardisierten Tabellendaten (z.B. mittels Komma oder Tabulator begrenzt u. ä.) oder mittels OCR-Programm gescannte Dokumente. Neu ist jetzt die integrierte Schnittstelle zum InternetExplorer, sodass einzelne Seiten oder

askSam

\title{
Das Schweizer Messer zur intelligenten Informationsbeschaffung
}

\author{
R. H. Bubenzer, Hamburg \\ NOTFALL \& HAUSARZTMEDIZIN 2005; 31: 301-302
}

ganze Websites auf Knopfdruck in eine leicht durchsuchbare lokale Datenbank überführt werden können. Wahlweise mit oder ohne Abbildungen oder Originalseiten (als Anhang). Zudem können Sie gleich beim Archivieren Kommentare und Schlüsselwörter mit angeben oder die Informationen kategorisieren.

Doch askSam würde nicht auch „Datenbank“ heißen, verhielte sich die Software nicht wie eine normale Datenbank. So können zum Beispiel nach Definition von Datenfeldern mit Typklassifikation Datensätze nach und nach mit standardisierten Daten (auch aus Praxis-Importdaten z.B. in BDT-Format) gefüllt werden. Noch besser: askSam erkennt auch scheinbar - unstrukturierte Daten, weist diese dann automatisch erkannten Datenfeldern zu. Übrigens auch bei Importen aus dem Internet. Die Altanwender von askSam, oft Juristen, Historiker, Journalisten oder auch Biowissenschaftler, schätzen von Anfang an diese Eigenschaft: Selbst bei Vorliegen einer strukturierten Datenbank können beliebige Felder jederzeit ergänzt und erweitert werden (eben „Freiform“...), es gibt keine Beschränkungen beim Umfang von Datensätzen.

Großartige Möglichkeiten zur Report-Generierung (strukturierte, formatierte Informationsausgabe), Export, Publikation von askSamDatenbank im Internet („askSam Publisher"), einfachste Erstellung auch von strukturierten Datenbanken, Multiuser-Nutzung im Netzwerk oder für viele Zwecke ausreichende Textverarbeitungsmöglichkeiten sind weitere Möglichkeiten der „eierlegenden Wollmilch-Sau“ askSam.

\section{askSam-Anwendungen \\ in der Praxis}

Einige der potentiellen Anwendungsmöglichkeiten von askSam im Praxisalltag:

Archivierung von Internetseiten

Seit Version 6.0 sind relevante Internetseiten bei ihrer Archivierung auch einzelnen Kategorien zuzuordnen und so zum Beispiel nach Fachgebiet, Indikation oder Therapiekonzept strukturierbar. Erst askSam macht - im Gegensatz zu allen dem Autor bekannten Produkten - den

\section{Informationen und Empfehlenswertes zu askSam}

Weitere Informationen zur Version 6.x (englisch)

Weitere Informationen zur Version 5.x (deutsch)

Forum - englisch

Forum - deutsch

Empfehlenswert: „MEMO5 - Fragen und Lösungen zu askSam 5.13 Pro“

vom 1.6.05 (672 KB) mit rund 250 Tipps und Tricks für Anfänger

und Fortgeschrittene

Infos über die akademischen Hintergründe von askSam

30-Tage funktionierende englische Vollversion von askSam 6 www.asksam.com/brochure.asp

www.asksam.de

www.asksam.com/forums/

ratman.at/ forum/index.php?act=SF\&f=69

flogehrke.homepage.t-online.de/memo5.zip

www.dlinfo.de/productdetails.php?cat=102\&show=asksam www.asksam.com/downloads/askSam.aspx 
Aufbau einer individuell skalierbaren medizinischen Fachdatenbank möglich, die auf die Bedürfnisse einer einzelnen Person oder einer einzelnen Praxis zugeschnitten sind.

\section{Abbildung von Teilbeständen medizinischer Datenbanken}

Da die Daten in medizinisch relevanten Datenbanken, z. B. MedLine, bereits strukturiert sind, gelingt der Aufbau einer eigenen askSam-Datenbank besonders leicht. Mit dem hier (www.asksam.com/ medline) vorgeschlagenen Ansatz übertrifft die Funktionalität jedes sonstige kommerzielle, auf Bibliographie-Archivierung oder Literaturzitation ausgerichtete Produkt. Unter anderem auch deshalb, weil die Anwender jeden Datensatz nach Bedarf erweitern können (neben Text auch mit Bildern, Grafiken oder Hyperlinks).

\section{Langzeit-Archivierung von E-Mail}

Abgesehen davon, dass alle EMail-Programme mit Dauer-Archivierung überfordert sind, fehlt auch die Möglichkeit einer so flexiblen Suche und Reportausgabe wie bei askSam. Mit den neueren Versionen können auch deutsche Outlook Express-Daten komplett und richtig übernommen werden.

\section{Zeitschriften-Sammlung}

Mit der jetzt vorhandenen PDFImportfunktion ist es ein Leichtes, Zeitschriften, die online oder per CD-

\section{Preise und Bezugsquellen}

\begin{tabular}{lll}
\hline Produkt & Preis & Bezugsquelle \\
\hline askSam Standard 6 (engl.) & $\$ 149,95$ & www.asksam.com \\
\hline $\begin{array}{l}\text { askSam Professional 6 } \\
\text { (engl., u. a. inkl. Zusatz-Indizierung, }\end{array}$ & $\$ 395,00$ & \\
$\begin{array}{l}\text { VBS-Programmierung, autom. Wortlisten) } \\
\text { askSam Professional 5.16 (deutsch), }\end{array}$ & $\begin{array}{l}\text { 396,69 EUR } \\
\text { deutsche Version 6 }\end{array}$ & www.asksam.de \\
$\begin{array}{l}\text { derzeit wird noch nicht ausgeliefert } \\
\begin{array}{l}\text { askSam Viewer (engl.) zur Anzeige von } \\
\text { Datenbanken ohne Veränderungsmöglichkeit }\end{array}\end{array}$ & kostenlos & $\begin{array}{l}\text { dl.filekicker.com/ send/ ile/ } \\
\text { 148126-3VF2/ asviewersetup.exe }\end{array}$ \\
\hline
\end{tabular}

ROM verfügbar sind, in eine ultraschnelle Datenbank zu überführen und auf diese Weise rasch verfügbar zu machen. Bei dem Import werden jedoch nur die Textinformationen in die Datenbank übertragen, viele Formateigenschaften gehen verloren. Wer die Dokumente im Original behalten und anschauen möchte, kann beim Import angeben, dass jedes Dokument zusätzlich im Original in den Datenbestand eingefügt wird (,save as attachment“). Zum Betrachten ist dann jedoch ein PDF-Viewer, zum Beispiel Acrobat Reader, nötig.

\section{Hinweis}

Alle hier geschilderten, qualitativ völlig unterschiedlichen Daten könnten im Bedarfsfall in einer einzigen askSam-Datei gespeichert werden. Dies ist mit der neuen Version jedoch nicht mehr unbedingt nötig, weil jetzt die gleichzeitige Suche über viele Dateien möglich ist.

\author{
Anschrift des Verfassers \\ Rainer H. Bubenzer (DJV, KdM) \\ Medizin- und Wissenschaftsjournalist \\ multi MED vision/presseteam volksdorf - \\ hamburger medizinredaktion \\ Borselstraße 9 \\ 22765 Hamburg \\ Tel: $040 / 41912873$ \\ Fax:040/41912877 \\ E-Mail: Rainer@Bubenzer.com
}

